\title{
Spin polarization in a two-dimensional electron gas modulated periodically by ferromagnetic and Schottky metal stripes
}

\author{
G. Papp,,$^{1,2}$ P. Vasilopoulos, ${ }^{3}$ and F. M. Peeters ${ }^{1}$ \\ ${ }^{1}$ Departement Fysica, Universiteit Antwerpen (Campus Drie Eiken), Universiteitsplein 1, B-2610 Antwerpen, Belgium \\ ${ }^{2}$ Department of Theoretical Physics, University of Szeged, Aradi vtk. tere 1, H-6720 Szeged, Hungary, \\ and Institute of Physics, University of West Hungary, Bajcsy Zs.út 5-7, H- 9400 Sopron, Hungary \\ ${ }^{3}$ Department of Physics, Concordia University, 1455 de Maisonneuve Quest, Montréal, Quebec, Canada H3G 1 M8
}

(Received 21 December 2004; published 14 September 2005)

\begin{abstract}
Spin-dependent electron transport in a two-dimensional electron gas, periodically modulated by parallel ferromagnetic and Schottky metal stripes, is studied theoretically. Assuming ballistic transport, the spindependent transmission, conductance, and polarization are evaluated for a range of experimentally accessible parameters. The structures of these quantities for one ferromagnetic-Schottky stripe unit can be significantly enhanced with increasing the number of units and highly polarized transport can be achieved. This structure is rounded off but survives to a good extent for finite temperatures of a few degrees. Results are given for parallel as well as antiparallel magnetizations of the ferromagnetic stripes between two consecutive units. In the latter a high degree of spin selection results and the spin polarization can be controlled very efficiently with Schottky metal stripes.
\end{abstract}

DOI: 10.1103/PhysRevB.72.115315

PACS number(s): 73.40.Gk, 72.25.Dc, 75.75.+a

\section{INTRODUCTION}

Recently the electronic transport properties of twodimensional electron gas (2DEG) in the presence of inhomogeneous magnetic fields have attracted considerable attention. For instance, the experimental realization of magnetic dots and antidots and the patterning of ferromagnetic (FM) materials integrated with semiconductors (SC) have created magnetic barriers (MB) or wells, periodic and quasiperiodic magnetic superlattices and motivated a considerable amount of research. ${ }^{1}$ The combination of FM and SC materials is realized by deposing an FM material on top of a near-surface 2DEG formed in modulation-doped semiconductor heterostructures. ${ }^{2}$ The FM material provides a magnetic field which influences locally the motion of the electrons in the semiconductor heterostructure. The effects caused by the MB system $^{3}$ are very different from the well-known potential barrier because the electron tunneling becomes a twodimensional (2D) problem. The transmission depends not only on the energy of the impeding electrons but also on the direction of their velocity toward the barrier. When both the charge and the spin of the electrons are taken into account, the electronic transport can become spin polarized. ${ }^{4-7}$ Several works have explored the possibility of producing spin memory devices ${ }^{8}$ or spin transistors, ${ }^{9}$ as well as exploiting the properties of spin coherence for quantum computation. $^{10,11}$ The feasibility of spin filtering in MB nanostructures has been studied and discussed extensively in the last decade. ${ }^{12-19}$ Very recently it was shown ${ }^{20}$ that the intrinsic symmetry in a single FM stripe structure can be broken and thus spin filtering can be achieved by placing a Schottky normal-metal (SM) stripe parallel to the FM stripe on top of the 2DEG. Considering such a structure as a single unit and repeating it periodically, spin-polarized transport can be enhanced significantly upon increasing the number of units. In addition, a good spin-selection control can be achieved by considering antiparallel magnetizations of the FM stripes between two consecutive units. Further, we provide some finite-temperature results and show that these effects survive for finite but low temperatures.

In Sec. II we present the formalism and in Sec. III the numerical results. Concluding remarks follow in Sec. IV.

\section{FORMALISM}

We consider a 2DEG in the $(x, y)$ plane modulated periodically by parallel FM and SM stripes placed on top of the structure. Two units of such a structure are schematically depicted in Fig. 1. The widths of the FM and SM stripes are, respectively, $d_{\mathrm{M}}$ and $d_{\mathrm{E}}, D$ is the spacing between these stripes, and $z_{0}$ the distance between them and the 2DEG. We assume that the magnetic field provided by the FM stripes $B_{z}(x)$ and the electrical potential induced by the Schottky stripes $U(x)$ are homogeneous in the $y$ direction and vary only along the $x$ axis in the manner shown in Figs. 1(b) or 1(d). The Hamiltonian describing such a system, in the single-particle, effective-mass approximation, is

$$
H=\frac{p_{x}^{2}}{2 m^{*}}+\frac{\left[p_{y}+e A_{y}(x)\right]^{2}}{2 m^{*}}+U(x)+\frac{e g^{*}}{2 m_{0}} \frac{\sigma \hbar}{2} B_{z}(x) .
$$

Here $m^{*}$ is the effective mass and $m_{0}$ the bare mass of the electron, $\left(p_{x}, p_{y}\right)$ the components of the electron momentum, and $g^{*}$ the effective Lande factor. Further, $\sigma$ is the electron spin pointing up $(\sigma=+1)$ or down $(\sigma=-1)$, and $A_{y}(x)$ is the component of magnetic vector potential given, in the Landau gauge, by $\mathbf{A}=\left[0, A_{y}(x), 0\right]$. Because the system is translational invariant along the $y$ direction the solution of the stationary Schrödinger equation $H \Psi(x, y)=E \Psi(x, y)$ can be sought in the form $\Psi(x, y)=\exp \left(i k_{y} y\right) \psi(x)$ with $\hbar k_{y}$ the expectation value of the momentum $p_{y}$ in the $y$ direction. The wave function $\psi(x)$ satisfies the following one-dimensional (1D) Schrödinger equation: 


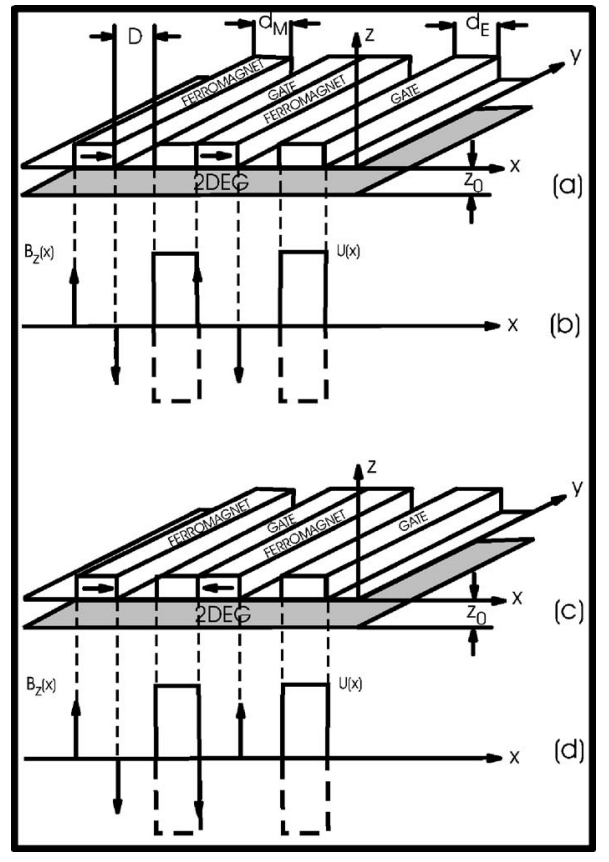

FIG. 1. (a) Schematic illustration of the structure. On top of a 2DEG, parallel FM and SM stripes are placed periodically as shown for only two units. The various parameters are defined in the text. The magnetization of all FM stripes is parallel to the $x$ axes. (b) The profile of the magnetic field $B_{z}(x)$ and of the electric potential $U(x)$ corresponding to (a). (c) As in (a) but with the magnetization of any two consecutive FM stripes antiparallel to each other. (d) The same as in (b) for the structure shown in (c).

$$
\begin{aligned}
& {\left[\frac{d^{2}}{d x^{2}}-\left(k_{y}+\frac{e A_{y}(x)}{\hbar}\right)^{2}\right.} \\
& \left.\quad+\frac{2 m^{*}}{\hbar^{2}}\left[E-U(x)-\frac{e g^{*}}{2 m_{0}} \frac{\sigma \hbar}{2} B_{z}(x)\right]\right] \psi(x)=0 .
\end{aligned}
$$

If we introduce the characteristic parameters: ${ }^{3} \omega_{0}=e B_{0} / m^{*}$ with $B_{0}$ a typical magnetic field, and $\ell_{B}^{2}=\hbar / e B_{0}$, the Schrödinger equation becomes

$$
\left[\frac{d^{2}}{d x^{2}}+2\left[E-V\left(x, k_{y}\right)\right]\right] \psi(x)=0,
$$

where all quantities are expressed in dimensionless units: $x$ $\rightarrow \ell_{B} x, E \rightarrow \hbar \omega_{c} E$ and

$$
V\left(x, k_{y}\right)=\left[k_{y}+A_{y}(x)\right]^{2} / 2+U(x)+g^{*} m^{*} \sigma B_{z}(x) / 4 m_{0},
$$

with $A_{y} \rightarrow B_{0} \ell_{B} A_{y}, B_{z}(x) \rightarrow B_{0} B_{z}(x)$ and $k_{y} \rightarrow k_{y} / \ell_{B}$. The problem is now reduced to a $1 \mathrm{D}$-tunneling problem. The $1 \mathrm{D}$ potential $V\left(x, k_{y}\right)$ depends on the wave vector $k_{y}$, the arrangement of stripes, and also on the interaction between the nonhomogeneous magnetic field and the electron spin. Matching the wave functions and their derivatives at all interfaces, the spin-dependent transmission probability through this system $T_{\sigma}\left(E, k_{y}\right)$, for electrons incident with energy $E$, wave vector $k_{y}$, and spin orientation $\sigma$ can be determined with the transfer matrix technique. ${ }^{21}$ Then one can evaluate the electron spin polarization defined by

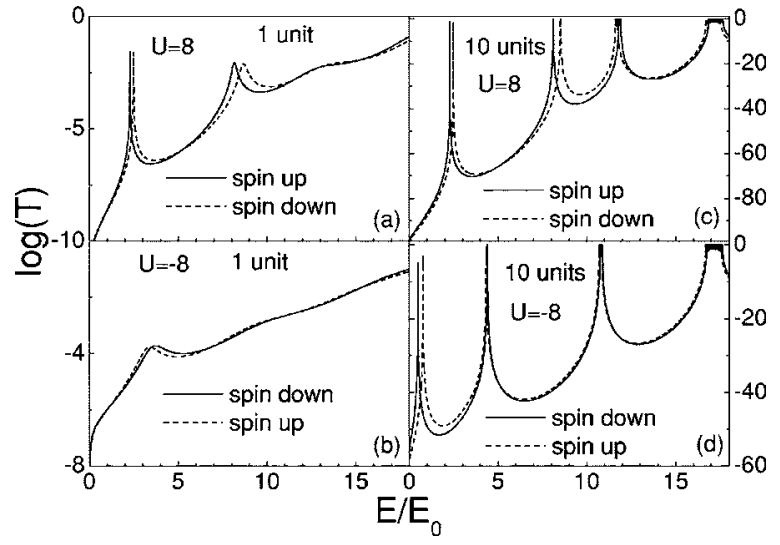

FIG. 2. Logarithm of spin-dependent transmission probability as a function of energy for one unit, with $U=8 \mathrm{in}$. (a) and $U=-8$ in. (b), and for ten units with $U=8$ in. (c) and $U=-8$ in. (d). The other parameters are $k_{y}=0, B=6$, and $d_{M}=D=d_{E}=1$.

$$
P\left(E, k_{y}\right)=\frac{T_{+}\left(E, k_{y}\right)-T_{-}\left(E, k_{y}\right)}{T_{+}\left(E, k_{y}\right)+T_{-}\left(E, k_{y}\right)} .
$$

In addition, one can evaluate the conductance $G$ as the electron flow averaged over half the Fermi surface ${ }^{22}$

$$
\begin{aligned}
G & =2 G_{0} \sum_{\sigma=-1,1} \int_{-\pi / 2}^{\pi / 2} T_{\sigma}\left(E_{F}, \sqrt{2 E_{F}} \sin \phi\right) \cos \phi d \phi \\
& =2 G_{0} \sum_{\sigma=-1,1} \int_{-1}^{1} T_{\sigma}\left(E_{F}, \sqrt{2 E_{F}} u\right) d u,
\end{aligned}
$$

where $\phi$ is the angle of incidence relative to the $x$ direction $G_{0}=e^{2} m^{*} v_{F} L_{y} / h^{2}$, where $L_{y}$ is the length of the structure in the $y$ direction and $v_{F}$ the Fermi velocity. ${ }^{23}$ The total conductance is the sum of the spin-up and spin-down conductances.

\section{NUMERICAL RESULTS}

The transmission probability and the polarization of transmitted electrons for different numbers of units have been calculated using the simplified magnetic field and electric potential profile shown in Figs. 1(b) and 1(d). The $\delta$-function magnetic field profile is an approximation for the real fringe fields of the ferromagnets and we assume that the effect of the parallel magnetic field component on the electron can be neglected. In previous works ${ }^{1,20}$ it was shown that such a $\delta$-function approximation is sufficiently accurate for obtaining qualitatively correct estimates for the tunneling properties. The material parameters used in the calculation were taken for InAs systems. Explicitly, we used $\mathrm{m}^{*}$ $=0.024 m_{0}, g^{*}=15$ and the estimate $B_{0}=0.1 \mathrm{~T}$ which is a realistic value. This leads to the units $\ell_{B}=813 \AA$, and $E_{0}$ $=\hbar \omega_{0}=0.48 \mathrm{meV}$. In Sec. III A we present results corresponding to the profile of Fig. 1(b) and in Sec. III B those for the profile of Fig. 1(d). 


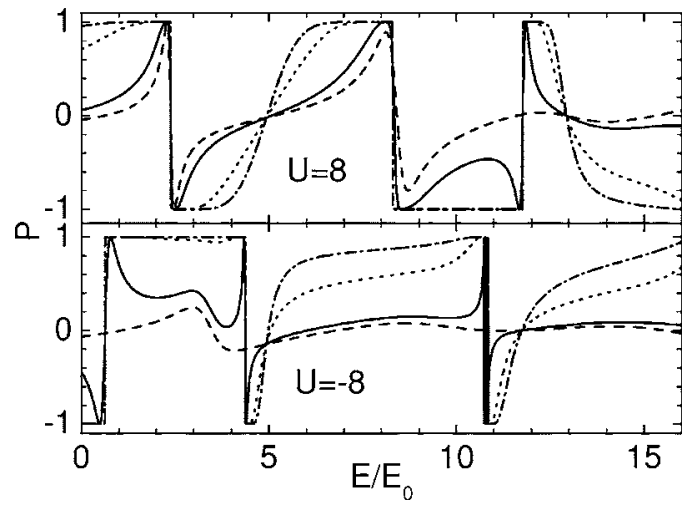

FIG. 3. Energy dependence of the spin-polarization $P$ for $U=8$ (top panel) and $U=-8$ (bottom panel). The dashed, solid, dotted, and dash-dotted curves are, respectively, for one, two, ten, and twenty units. The parameters are those of Fig. 2.

\section{A. Parallel magnetizations}

In Fig. 2 we plot the spin-up and the spin-down transmission probabilities as a function of the energy for $B=6$ (the strength of $B_{z}$ ) and $d_{\mathrm{M}}=D=d_{\mathrm{E}}=1$ with $U=8$ (upper panels) or $U=-8$ (lower panels) and the indicated number of units. For one unit the transmission for $U=-8$ is very different from that for $U=8$. The reason is that for positive $U$ the transmission relies on spin-dependence resonant tunneling through quasibound states of an asymmetrical double barrier well system, while for negative $U$ the tunneling is through the virtual states produced by the above potential well. For negative $U$ at least one more FM stripe is necessary to assure the possibility of resonant tunneling. The presence of the Zeeman term alters the transmission probability for all values of energy. For positive $U$ the resonant peaks shift to higher energy for the spin-down electrons, and to lower energy for the spin-up electrons but this shift is nonsymmetric. Increasing the number of units leads to more pronounced resonance peaks for positive or negative $U$. In the latter case though the shifting of the peaks depends on their position. The first resonance is shifted to higher energy for spin-up electrons, and lower energy for spin-down electrons relative to the corresponding one when the Zeeman term is neglected. Each peak consists of $n-1$ peaks, where $n$ is the number of units, due to the dispersion determined by the imposed periodicity and the coupling among successive quantum wells [Fig. 2 (right panels)].

The electron spin polarization $P$, given by Eq. (5), is shown in Fig. 3. The results for 1, 2, 10, and 20 units are shown, respectively, by the dashed, solid, dotted, and dashdotted curves for $U=8$ (top panel) and $U=-8$ (bottom panel). For one unit one can see that the polarization changes sign when the electron energy varies between the values that give the resonances for both spin orientations. The change of $P$ for two units is small for positive $U$ because the transmission (not shown) does not change significantly. This change is rather significant for negative $U$ because of the possibility of resonant tunneling: The potential contains an asymmetric double barrier-well system. The polarization of transmitted beams can reach $100 \%$.

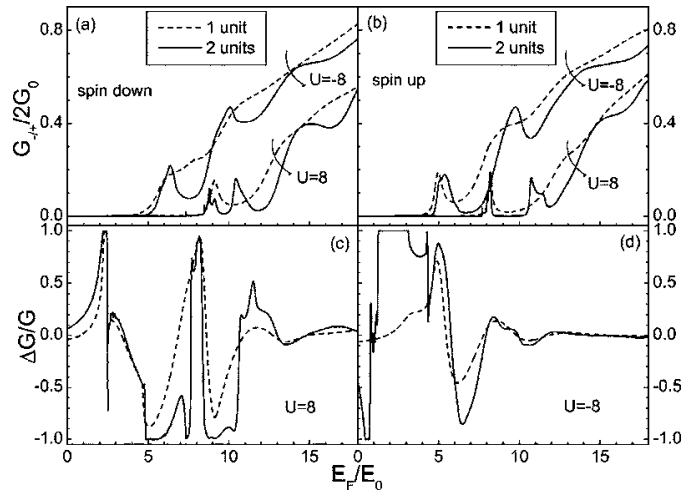

FIG. 4. Conductance versus Fermi energy for spin-down (a) and spin-up (b) electrons and two different electric barrier heights $U$ $= \pm 8$. Panels (c) and (d) show the relative spin-conductance excess versus Fermi energy for $U=8$ in. (c) and $U=-8$ in. (d). The dashed and solid curves are for one and two units, respectively.

Having seen the transmission and polarization results, one may wonder to what extent their structure is reflected in measurable quantities, which involve some kind of averaging, such as the conductance $G$ given by Eq. (6). Figures 4(a) and 4(b) show the conductances versus Fermi energy for spin down (a) and spin up (b) and two electric barrier heights $U$ $= \pm 8$, with one unit (dashed curves) and with two units (solid curves). The two conductances generally decrease when the number of units is increased except at the resonance positions. The reason is that the transmission probability peaks are sensitive to the width of the potential barriers, they are sharper when the barriers become wider. By increasing the number of units the total width of the barriers increases as well and the conductance, which is determined by the transmission probability, generally decreases. As a consequence, the peak-to-valley ratio increased. For example, for $U=-8$ around $5 E_{F} / E_{0}$ these ratios are 2.97 and 11.2 for spin-down and spin-up electrons, respectively, while it is clearly seen that there is no valley at all for spin-down electrons when only one unit is present. To investigate the spin current transport (i.e., $\Delta G=G_{+}-G_{-}$) versus the charge transport (i.e., $G$ $\left.=G_{+}+G_{-}\right)$, the quantity $\Delta G / G=\left(G_{+}-G_{-}\right) /\left(G_{+}+G_{-}\right)$was calculated. Here $G_{+}$and $G_{-}$are the conductances with electron spin up and spin down, respectively. This quantity ${ }^{14,24}$ expresses the relative spin-conductance excess at the Fermi energy, i.e., for zero temperature. Panels (c) and (d) show the relative spin-conductance excess versus the Fermi energy for $U=8$ (c) and for $U=-8$ (d) with one unit (dashed curves) and with two units (solid curves). It is found that $\Delta G / G$ increases (decreases) with the Fermi energy in the low-energy region for positive $U$ (negative $U$ ) and it reaches $\sim 100 \%$ even with a doubling of the number of units both for positive and negative $U$, although the corresponding conductances in the energy region are rather small. This behavior can be understood by inspection of the transmissions shown in Fig. 2 (c); there it is clearly seen that in the low energy region new resonances appear for $U=-8$. When the Fermi energy varies from the resonant peak to the adjacent valley, $\Delta G / G$. is switched from positive to negative values for both negative and positive $U$ values.

Here we should notice that there are two other possibilities to repeat the unit: The first unit with positive $U$ value 


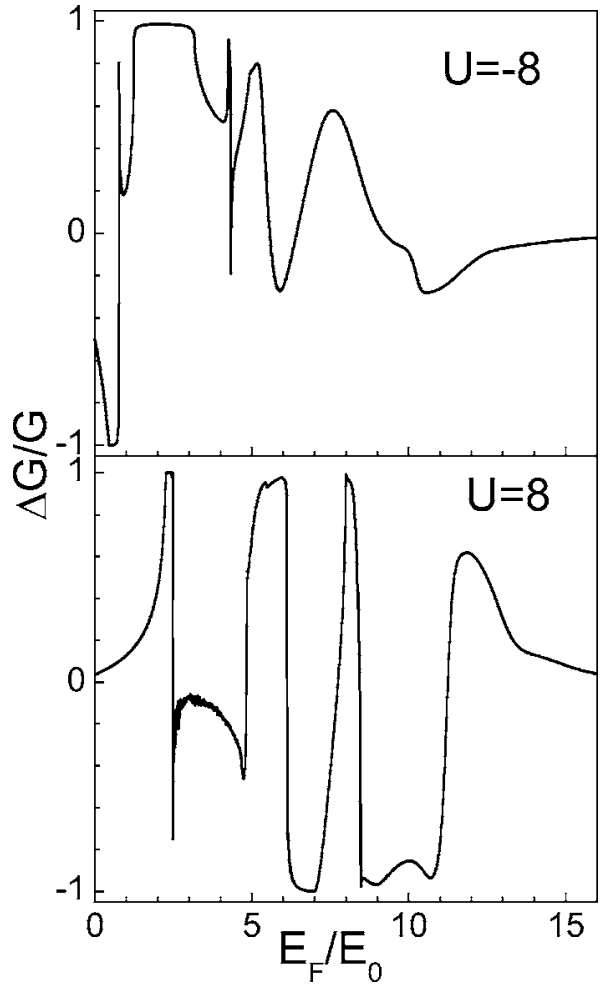

FIG. 5. Relative spin-conductance excess as a function of the Fermi energy for $U=-8$ (upper panel) and $U=8$ (lower panel) with $d_{M}=D=d_{E}=1$ in the first unit and $d_{M}=D=0.5, d_{E}=1$ in the second one.

and the second with a negative value of $U$ or vice versa. The behavior of the transmission or conductance will be different than those discussed above. Of course, the behavior of the system depends on the geometrical arrangement of the stripes, too. In Fig. 5 we show the relative spin-conductance excess versus Fermi energy, when the second unit contains a layer with a smaller width, e.g., $d_{\mathrm{M}}=D=0.5$. While for $U=-8$ (upper panel) the change of $\Delta G / G$ is small, the variation in case of $U=8$ (lower panel) is much larger. It should be emphasized that the intrinsic symmetry of the FM barriers holds independent of their number, and in order to have spin polarization the potential barriers are necessary. Figure 6 shows the spin-down and spin-up conductances (upper panels) as well as the relative spin-conductance excess (lower panels) versus Fermi energy for 20 units with $U=8$ (left panels) and $U=-8$ (right panels). We see again a considerable structure in $\Delta G / G$ brought about by the increased number of identical units whereas in Fig. 5 this structure resulted from the different sizes of the two units involved.

To generalize our results to nonzero temperature ${ }^{21,23}$ we have to replace any function of $G\left(E_{F}\right)$, which depends on the Fermi energy $E_{F}$, by the corresponding average over the derivative of the Fermi function: $G(\mu)=\int d \varepsilon G(\varepsilon)\left(-\partial f_{0} / \partial \varepsilon\right)$, where $f_{0}=\left\{\exp \left[(\varepsilon-\mu) / k_{B} T\right]+1\right\}^{-1}$ and $\mu$ is the chemical potential. Figure 7 shows the spin polarization at three different temperatures for $U=-8$ (upper panel) and $U=8$ (lower panel). The number of units is 20 . As can be seen, finite temperatures smoothen the zero-temperature results, e.g., of Fig. 6 but a significant structure remains, especially for $U$ $=8$, up to $T=4 \mathrm{~K}$.

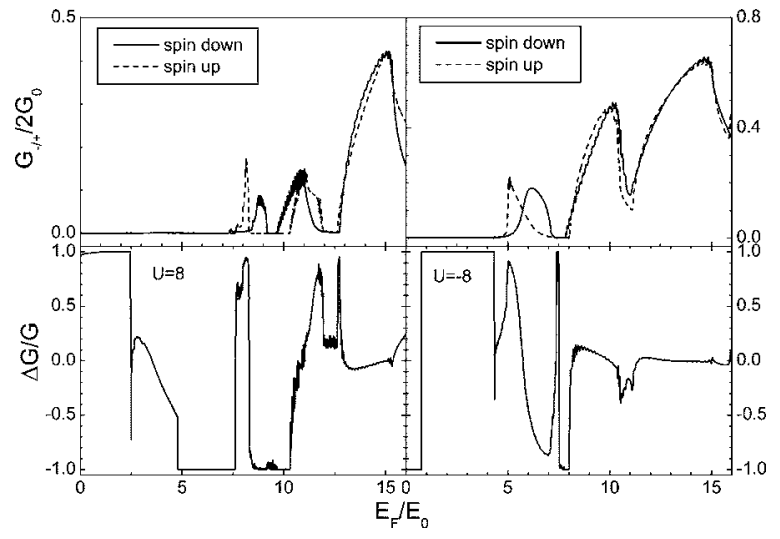

FIG. 6. Conductance and relative spin-conductance excess as a function of Fermi energy for 20 units, $U=8$ (left panels) and $U$ $=-8$ (right panels), $B=6$, and $d_{M}=D=d_{E}=1$.

\section{B. Antiparallel magnetizations}

Another method to realize wave vector filtering and spin polarization in a $2 \mathrm{DEG}$, using only antisymmetric doublebarrier magnetic structures, was reported in Ref. 18. The relevant magnetic field profile is shown in Fig. 1(d) and the magnetizations of any two consecutive FM stripes are antiparallel to each other. In such a system the potential $U(x)$ is absent, i.e., $d_{E}=0$. The resulting potential $V\left(x, k_{y}\right)$ has an even symmetry with respect to $k_{y}$ about the center. As we know, for particles traversing a potential in opposite directions the transmission is always equal. Therefore, such

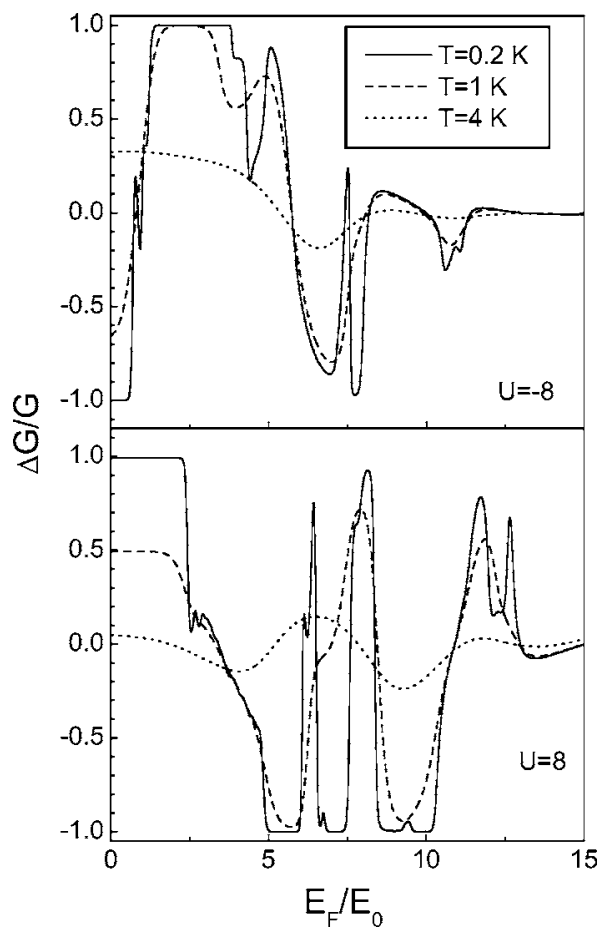

FIG. 7. Relative spin-conductance excess, through 20 units, as a function of energy for finite temperatures. The solid, dashed, and dotted curves are for $T=0.2 \mathrm{~K}, T=1 \mathrm{~K}$, and $T=4 \mathrm{~K}$, respectively. The other parameters are $B=6$, and $d_{M}=D=d_{E}=1$. 


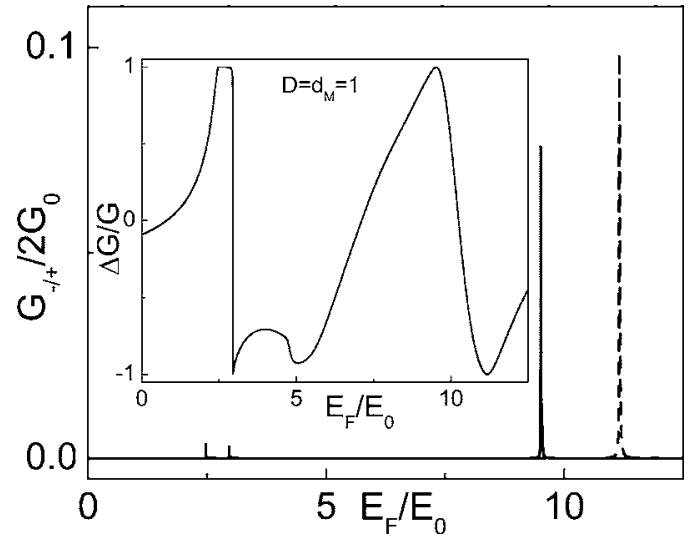

FIG. 8. Conductance versus the Fermi energy for spin-down (solid curve) and spin-up (dashed curve) electrons for asymmetric double magnetic barriers with $D=d_{M}=1$ and $B=6$. In the inset the relative spin-conductance excess is shown as a function of the Fermi energy.

symmetry results in the invariance of the transmission with respect to the replacement $k_{y} \rightarrow-k_{y}$. It is thus sufficient to take only positive $k_{y}$ values into account. For such a system the resonant peaks, relative to those with parallel magnetizations, shift to higher energy for spin-down electrons, and to lower energy for the spin-up electrons. The resonance peaks are extremely sharp, so $T_{-}$nearly vanishes for those energies where $T_{+}$is in resonance and vice versa. Therefore the polarization of transmitted electrons can reach $100 \%$. The resonance peaks for both spin-up and spin-down electrons shift to lower energy by increasing the separation $D$ or by decreasing the magnetic barrier intensity. This sharpness appears in measurable quantities also, as clearly seen in Fig. 8, where the spin-up $\left(G_{+}\right.$, solid curve) and spin-down $\left(G_{-}\right.$, dashed curve) conductances are shown. The inset shows the relative spin-conductance excess versus the Fermi energy, for InAs with $B=6, D=d_{M}=1$. It is found that the $\Delta G / G$ increases with Fermi energy in the low-energy region and it reaches nearly $100 \%$ at resonant energy for $\sigma=+1$ and remains almost at this value until the resonant energy for $\sigma=$ -1 is reached, despite the averaging of the transmission probability over half the Fermi surface. For Fermi energies that are higher than the latter resonant energy, the relative spin-conductance excess remains negative in this range in which $T_{-}$dominates. Further raising the Fermi energy makes $\Delta G / G$ increase and reach about $100 \%$.

To assess the effect of breaking the symmetry of the antisymmetric double-barrier magnetic system with SM stripes, we have numerically calculated the $\Delta G / G$ for the system depicted in Fig. 1(d). Figure 9 shows the relative spinconductance excess for asymmetric double-barrier magnetic barrier system, i.e., two units of FM and SM stripes with antiparallel magnetization of the FM stripes $B=6$ and $U=$ -8 . The dashed curve is for $D=2 ; d_{M}=1$ and the solid one for $D=d_{M}=d_{E}=1$. It is clearly seen that the spin polarization can be changed efficiently with the SM stripes and results in an approximate periodic behavior of $\Delta G / G$. The results are

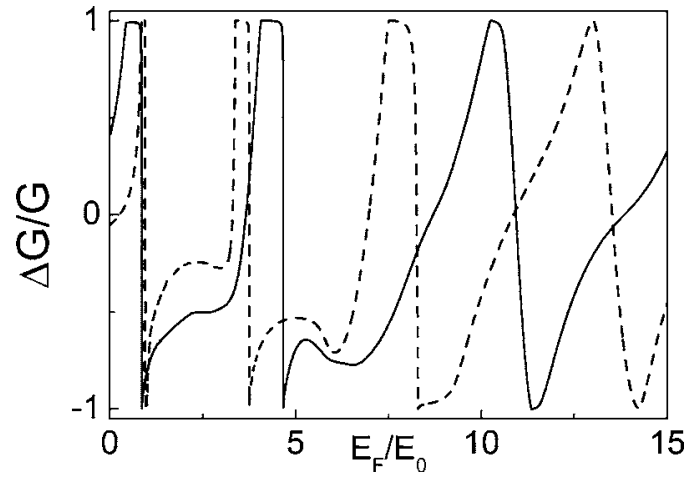

FIG. 9. Relative spin-conductance excess as a function of the Fermi energy for asymmetric double magnetic barriers: Two units of FM and SM stripes with antiparallel magnetization of the FM stripes and $B=6$ and $U=-8$. The dashed curve is for $D=2, d_{M}=1$ and the solid one for $D=d_{M}=d_{E}=1$.

similar to those obtained for the transmission through a waveguide, as a function of its length, in the presence of spin-orbit interaction. ${ }^{25}$

\section{CONCLUDING REMARKS}

We studied spin-polarized, ballistic electron transport in a two-dimensional electron gas periodically modulated by ferromagnetic and Schottky metal stripes. The spin-dependent transmission, conductance, and polarization show a considerable structure as a function of the incident electron (Fermi) energy and of the various parameters that characterize the periodically repeated unit. This structure is significantly enhanced with increasing number of units and shows that a highly polarized transport can be achieved. In some cases the polarization acquires an approximate square-wave character as a function of the Fermi energy, cf. Fig. 3, lower panel. The relative spin-conductance excess exhibits a considerable structure, cf. Fig. 5, for positively and negatively charged Schottky metal stripes although the structure has only two units. In general, the structure in all these quantities is rounded off at finite temperatures but survives up to a few degrees, cf. Fig. 7.

We presented results for parallel as well as antiparallel magnetizations of the ferromagnetic stripes between two consecutive units. As Figs. 8 and 9 demonstrate, in the latter case a high degree of spin selection results and the spin polarization can be controlled very efficiently with Schottky metal stripes which break the symmetry of the potential created by the magnetic barriers.

We are not aware of any transport experiments involving the structures we studied. We hope that our findings, especially those for antiparallel magnetizations, will motivate appropriate experiments.

\section{ACKNOWLEDGMENTS}

This work was supported by the Flemish-Hungarian bilateral programme, the Canadian NSERC Grant No. OGP0121756, and by the Flemish Science Foundation (FWO-V1). 
${ }^{1}$ For a recent review, see e.g., F. M. Peeters and J. De Boeck, in Handbook of Nanostructured Materials and Nanotechnology, Vol. 3, edited by H. S. Nalwa (Academic Press, N.Y., 1999), p. 345 .

${ }^{2}$ V. Kubrak, F. Rahman, B. L. Gallagher, P. C. Main, M. Henini, C. H. Marrows, and M. A. Howson, Appl. Phys. Lett. 74, 2507 (1999); T. Vancura, T. Ihn, S. Broderick, K. Ensslin, W. Wegscheider, and M. Bichler, Phys. Rev. B 62, 5074 (2000).

${ }^{3}$ F. M. Peeters and A. Matulis, Phys. Rev. B 48, 15166 (1993); A. Matulis, F. M. Peeters, and P. Vasilopoulos, Phys. Rev. Lett. 72, 1518 (1994); G. Papp and F. M. Peeters, Phys. Status Solidi B 225, 433 (2001).

${ }^{4}$ A. Voskoboynikov, S. S. Liu, and C. P. Lee, Phys. Rev. B 59, 12514 (1999); A. Voskoboynikov, S. S. Liu, and C. P. Lee, ibid. 58, 15397 (1998); M. Sharma, S. X. Wang, and J. H. Nickel, Phys. Rev. Lett. 82, 616 (1999).

${ }^{5}$ P. Pfeffer and W. Zawadzki, Phys. Rev. B 59, R5312 (1999).

${ }^{6}$ E. A. de Andrada e Silva and G. C. La Rocca, Phys. Rev. B 59, R15583 (1999).

${ }^{7}$ L. Via, J. Phys.: Condens. Matter 11, 5929 (1999).

${ }^{8}$ J. M. Kikkawa, I. P. Smorchkova, N. Samarth, and D. D. Awschalom, Science 277, 1284 (1997).

${ }^{9}$ D. J. Monsma, R. Vlutters, and J. C. Lodder, Science 281, 407 (1998); X. F. Wang, P. Vasilopoulos, and F. M. Peeters, Appl. Phys. Lett. 80, 1400 (2002).

${ }^{10}$ D. P. DiVincenzo, Science 270, 255 (1995); D. Loss and D. P. DiVincenzo, Phys. Rev. A 57, 120 (1998); G. Burkard, D. Loss, and D. P. DiVincenzo, Phys. Rev. B 59, 2070 (1998).
${ }^{11}$ B. E. Kane, Nature (London) 393, 133 (1998).

${ }^{12}$ V. N. Dobrovolsky, D. I. Sheka, and B. V. Chernyachuk, Surf. Sci. 397, 333 (1998).

${ }^{13}$ Y. Guo, B. L. Gu, Z. Zeng, J. Z. Yu, and Y. Kawazoe, Phys. Rev. B 62, 2635 (2000).

${ }^{14}$ G. Papp and F. M. Peeters, Appl. Phys. Lett. 78, 2184 (2001); 79, 3198 (2001).

${ }^{15}$ H. Z. Xu and Y. Okada, Appl. Phys. Lett. 79, 3119 (2001).

${ }^{16}$ Y. Jiang and M. B. A. Jalil, Phys. Status Solidi B 235, 157 (2003); G. Papp and F. M. Peeters, ibid. 241, 222 (2004).

${ }^{17}$ Y. Guo, Z. Feng, B. L. Gu, and Y. Kawazoe, Phys. Rev. B 66, 045312 (2002).

${ }^{18}$ H. Z. Xu and Z. Shi, Appl. Phys. Lett. 81, 691 (2002); G. Papp and F. M. Peeters, ibid. 82, 3570 (2003); Y. Guo, J. H. Qin, X. Y. Chen, and B. L. Gu, Chin. Phys. Lett. 20, 1124 (2003).

${ }^{19}$ X. F. Wang and P. Vasilopoulos, Appl. Phys. Lett. 81, 1636 (2002).

${ }^{20}$ F. Zhai, H. Q. Xu, and Y. Guo, Phys. Rev. B 70, 085308 (2004).

${ }^{21}$ A. MacKinnon, Z. Phys. B: Condens. Matter 59, 385 (1985); G. Papp and F. M. Peeters, J. Phys.: Condens. Matter 16, 8275 (2004); Physica E (Amsterdam) 25, 335 (2005).

${ }^{22}$ M. Büttiker, Phys. Rev. Lett. 57, 1761 (1986).

${ }^{23}$ A. Matulis and F. M. Peeters, Phys. Rev. B 62, 91 (2000); The expression of $\mathrm{G}_{0}$ in Eq. (6) of Ref. 3 (b) should be divided by $2 \pi^{2}$.

${ }^{24}$ R. Benjamin and C. Benjamin, Phys. Rev. B 69, 085318 (2004).

${ }^{25}$ S. Datta and B. Das, Appl. Phys. Lett. 56, 665 (1990). 